\title{
Searching for Extrasolar Planets from UNSW
}

\author{
Jessie L. Christiansen ${ }^{1}$, M.C.B. Ashley ${ }^{1}$, M.G. Hidas ${ }^{1}$, \\ J.K. Webb ${ }^{1}$, A. Phillips ${ }^{1}$, M. Irwin ${ }^{2}$ and J. Irwin ${ }^{2}$ \\ ${ }^{1}$ Department of Astrophysics, University of New South Wales, Sydney, NSW 2052, Australia \\ email: jessiec@phys.unsw.edu.au \\ ${ }^{2}$ Institute of Astronomy, University of Cambridge
}

\begin{abstract}
The Automated Patrol Telescope, operated by the University of New South Wales, has been undertaking a search for extrasolar planets using the transit method. We present lightcurves from two recent promising candidates; spectroscopic follow-up using the ANU 2.3m telescope shows that the companions are probably low mass stars rather than planets, although more data will be needed to be certain. Additionally, we outline future improvements to our transit search: a hardware upgrade scheduled for 2006, and the addition of a robust trend-filtering algorithm to the data reduction software.
\end{abstract}

Keywords. extrasolar planets, transit, spectroscopic follow-up, trend-filtering.

\section{Latest Results}

The Automated Patrol Telescope is a $50-\mathrm{cm}$ optical telescope sited at Siding Spring Observatory (SSO), NSW, Australia. It is dedicated to an Extrasolar Planet Search, the details of which can be found in Hidas et al. (2005). From February to March 2005 we observed a pair of fields centred on $12 \mathrm{~h} \mathrm{RA},-36^{\circ}$ dec, obtaining 22 nights of good data (RMS $<4$ mmag for stars brighter than 10th magnitude). Lightcurves for close to 3000 stars brighter than 13th magnitude were compiled, and two promising candidates designated UNSW-TR-13 and UNSW-TR-14 were discovered.

UNSW-TR-13 displayed 2 complete and 3 partial transits, with a depth of 30mmag and a period of 2.283 days (Figure 1a). UNSW-TR-14 displayed 7 total and 3 partial transits, with a depth of $20 \mathrm{mmag}$ and a period of 0.640 days (Figure $1 \mathrm{~b}$ ). For comparison, Figure 1c shows a transit of TrES-1, the first transiting planet discovered with a small, wide field-of-view telescope (Alonso et al. 2004). It is clear that the APT is reaching the required level of precision to detect transiting extrasolar planets.

Time and weather constraints resulted in a failure to capture our candidates in transit with the ANU 40-inch telescope at SSO. However, we were able to obtain follow up observations with the DBS instrument on the ANU 2.3m telescope. Spectral classification of the candidate host stars and radial velocity curves are shown in Figure 2. These curves are constrained with the period and phase data taken from the lightcurves. The characteristics derived from the spectra are summarised in Table 1. UNSW-TR-13 is found to probably be orbited by an M dwarf, and UNSW-TR-14 appears to have a brown dwarf companion.

\section{Hardware Improvements}

With the assistance of a grant from the Australian Research Council, a new CCD camera has been commissioned for the APT, for installation in 2006. Table 2 shows a 

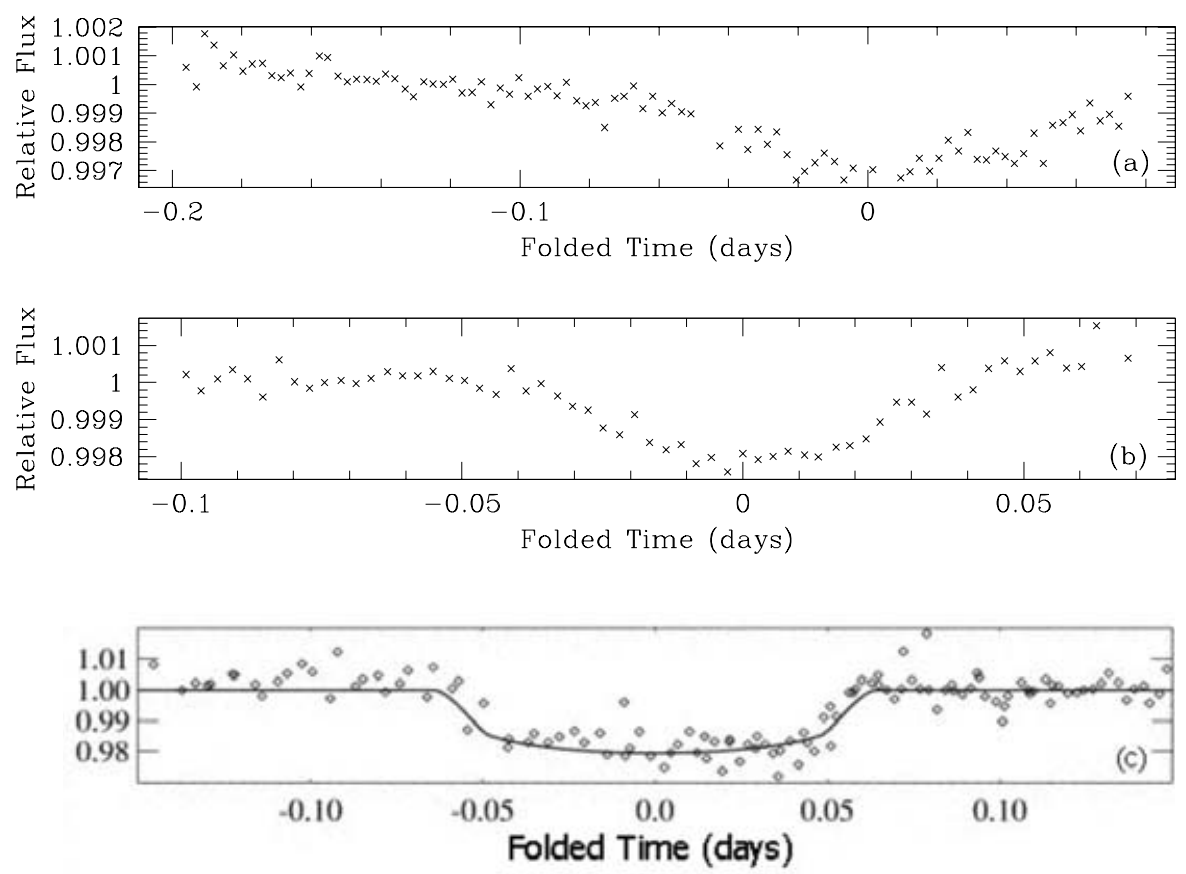

Figure 1. Panel (a) shows a sample transit from the lightcurve of UNSW-TR-13, panel (b) shows a sample transit from UNSW-TR-14 and for comparison, panel (c) shows a transit (taken with the $10 \mathrm{~cm}$ PSST instrument) of TrES-1, the first planet to be discovered with the transit method (Alonso et al. 2004).

Table 1 . The derived characteristics of the planetary candidate systems, where $\mathrm{K}$ is the relative radial velocity amplitude. We find that UNSW-TR-13 is orbited by a late-type M dwarf; for the quoted mass the orbital distance is $0.034 \mathrm{AU}$. UNSW-TR-14 is probably orbited by a brown dwarf companion at a distance of $0.015 \mathrm{AU}$; for the quoted mass. It should be noted that the lower mass limit of the UNSW-TR-14 companion is only $8.5 M_{\mathrm{Jup}}$ and we will be investigating this candidate further.

\begin{tabular}{|c|c|c|c|c|c|c|}
\hline Target & Sp. Type & I Magnitude & $\mathrm{M}_{1}\left(M_{\odot}\right)$ & $\mathrm{M}_{2}\left(M_{\odot}\right)$ & $\mathrm{K}\left(\mathrm{km} \mathrm{s}^{-1}\right)$ & Period (days) \\
\hline UNSW-TR-13 & G5V & 10.7 & 0.95 & $0.11 \pm 0.05$ & $17 \pm 8$ & 2.283 \\
\hline UNSW-TR-14 & $\mathrm{G} 2 \mathrm{~V}$ & 9.6 & 1.00 & $0.033 \pm 0.025$ & $8 \pm 6$ & 0.640 \\
\hline
\end{tabular}

comparison of the properties of the current CCD with the new CCD. Due to the increased number of data points, as a result of the larger sky coverage and improved time-sampling (the new CCD quantum efficiency will be three times higher than old, resulting in reduced exposure times), we expect to increase our chances of detecting planets at the current level of precision by a factor of nearly 5 . In addition, we would also expect to see higher precision photometry from the reduced undersampling, which would significantly increase our chances of detecting smaller amplitude transits. 

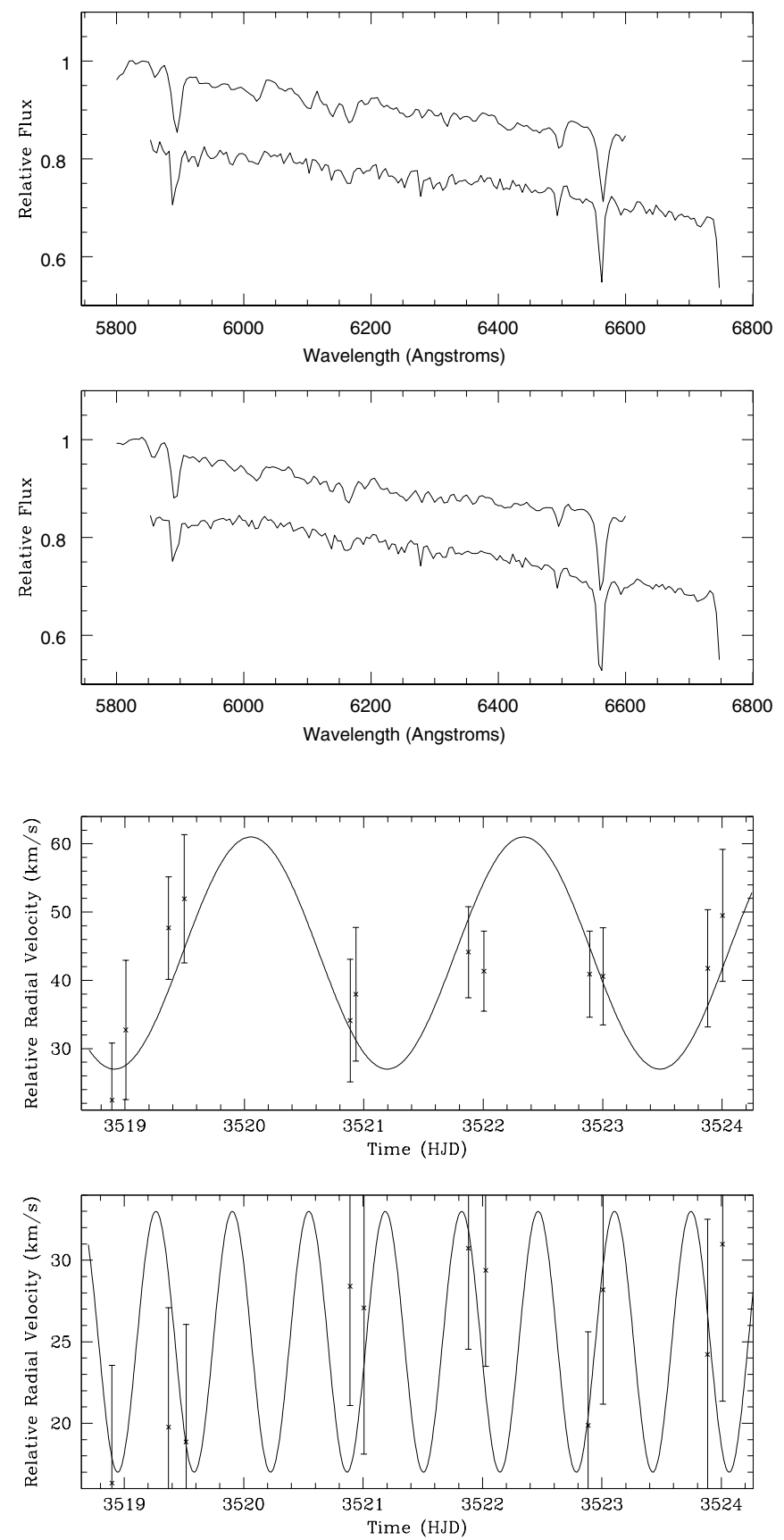

Figure 2. Follow-up results for the two UNSW candidates. The top two panels show data for object UNSW-TR-13. The host star has an I magnitude of 10.7; the stellar spectrum is shown in the left panel, with a model G5V spectrum offset above for comparison. On the right is the derived radial velocity curve with an amplitude of $17 \mathrm{~km} / \mathrm{s}$ and a period of 2.283 days. The bottom panels show the data for object UNSW-TR-14. The host star has an I magnitude of 9.6. Again, the spectrum is on the left, with a G2V model spectrum offset above, and its radial velocity curve, with an amplitude of $8 \mathrm{~km} / \mathrm{s}$ and a period of 0.640 days, is on the right. The original stellar spectra have been rebinned to $10 \AA$ for comparison with the model spectra, taken from the UVILIB spectral library (Pickles 1998). 
Table 2. The attributes of the current CCD being used in the APT are compared to the specified characteristics of the new CCD which is being built for the APT. ${ }^{(a)}$ This is the typical number of stars brighter than $13^{\text {th }}$ magnitude in a field at $b=15^{\circ} .{ }^{(b)}$ The number of images taken per hour. ${ }^{(c)}$ Although this CCD gives a coverage of 7 by 7 degrees, due to optical limitations the usable field of view of the CCD has a diameter of about 6 degrees.

\begin{tabular}{|c|c|c|}
\hline Attribute & Old CCD & New CCD \\
\hline CCD Size (pixels) & $1152 \times 770$ & $6 \mathrm{k} \times 6 \mathrm{k}$ \\
\hline Pixel Size $(\mu \mathrm{m})$ & 22.5 & 10 \\
\hline Image Scale & $9.43^{\prime \prime} /$ pixel & $4.19^{\prime \prime} /$ pixel \\
\hline Field of View & $2^{\circ} \times 3^{\circ}$ & $7^{\circ} \times 7^{\circ}(\mathrm{c})$ \\
\hline No. of $\operatorname{Stars}^{(a)}$ & $\sim 5000$ & $\sim 16000$ \\
\hline Cadence $^{(b)}$ & $\sim 30$ & $\sim 60$ \\
\hline
\end{tabular}

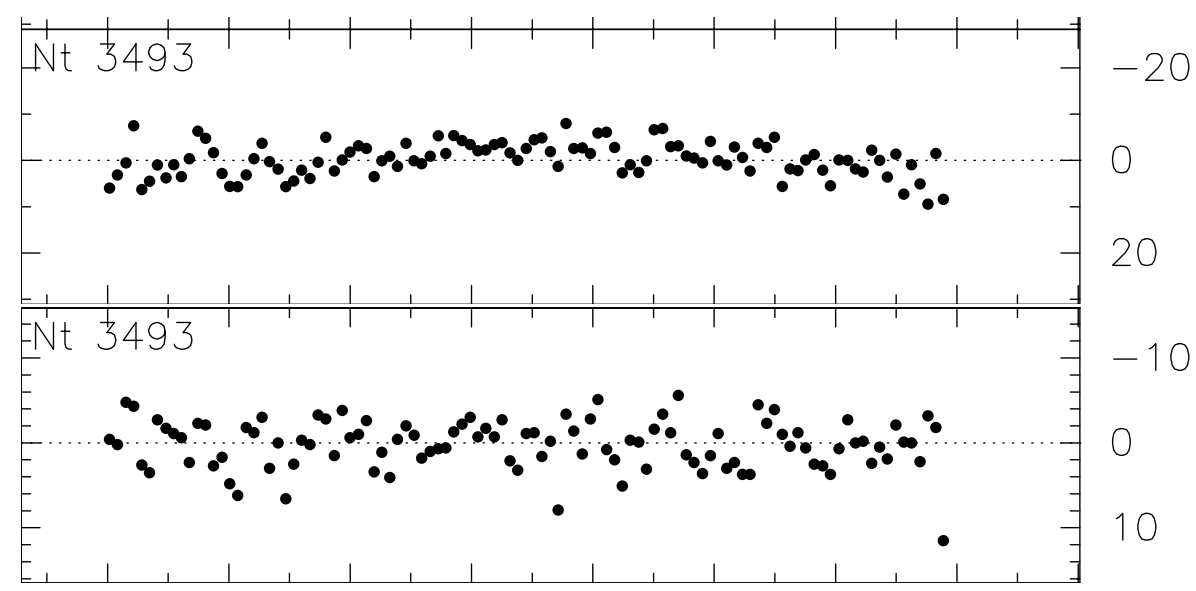

Figure 3. An example of the types of corrections handled by the trend-filtering algorithm. The top panel shows the original lightcurve, and the bottom panel shows the corrected lightcurve.

\section{Software Improvements}

We are currently developing a trend-filtering algorithm based on one described by Kovács et al. (2005) to remove residual systematics from our data. It is generally easy to gauge with the eye which signals are real and which are systematics. However, implementing a period-finding program to detect low amplitude transits when there are significant residual systematics is very inefficient. It generally leads to a high number of spurious detections with periods around $n$ days, where $n$ is a small integer. The basic operation of the algorithm is to use a subset of the stellar lightcurves as templates for construction of the lightcurves of the remaining stars. By subtracting a fitted linear combination of the template lightcurves from the original lightcurves, any systematics common to the stars and the template group should be theoretically removed. Although we are still implementing the final algorithm, preliminary results show a dramatic reduction in systematics, accompanied by a slight increase in the RMS noise, as shown in Figure 3 .

\section{Conclusions}

We are confident that the APT is reaching the precision necessary to detect transiting hot Jupiters, demonstrated by the sample transits presented here. We believe that with 
the addition of new hardware and software, our chances of detecting these planets will increase by at least a factor of 5 , and probably closer to a factor of 10 .

\section{Acknowledgements}

The authors thank Aliz Derekas for assistance with the ANU 2.3m observations.

\section{References}

Alonso, R., Brown, T.M., Torres, G., Latham, D.W., Sozzetti, A., Mandushev, G., Belmonte, J.A., Charbonneau, D., Deeg, H.J., Dunham, E.W., O'Donovan, F.T., \& Stefanik, R.P. 2005, Ap.J. 613, L153

Hidas, M.G, Ashley, M.C.B., Webb, J.K., Irwin, M., Phillips, A., Toyozumi, H., Derekas, A., Christiansen, J.L., Nutto, C., \& Crothers, S. 2005, MNRAS 360, 703

Kovács, G., Bakos, G., \& Noyes, R.W. 2005, MNRAS 356, 557

Pickles, A.J. 1998, PASP 110, 863 

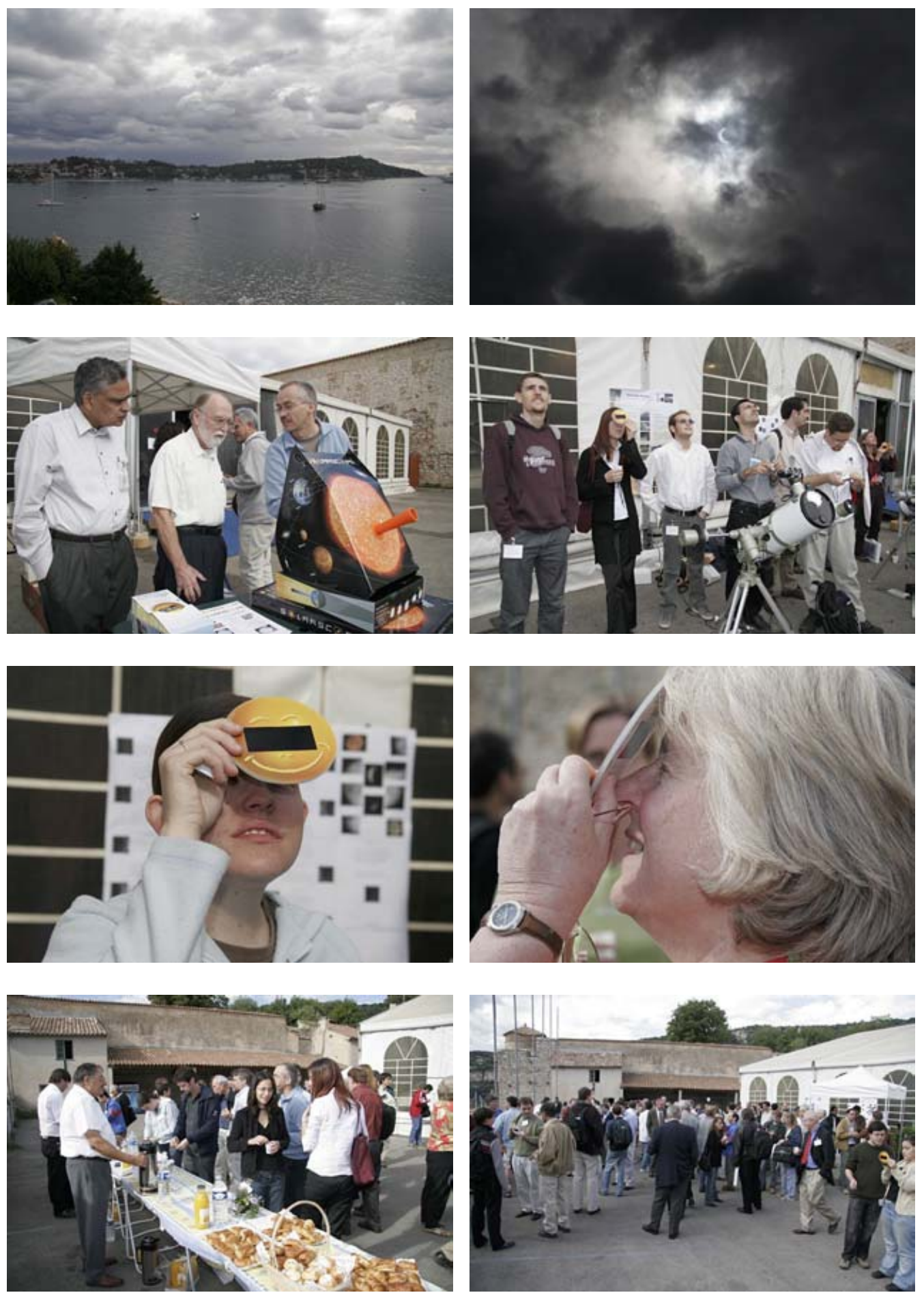

All photographs: Laurent Thareau [1.thareau@free.fr]. 